\title{
Intratumoral hemorrhage-related differences in the expression of vascular endothelial growth factor, basic fibroblast growth factor and thioredoxin reductase 1 in human glioblastoma
}

\author{
BULENT KAYA $^{1}$, ONUR ÇIÇEK ${ }^{2}$, FATIH ERDI ${ }^{1}$, SIDDIKA FINDIK $^{3}$, \\ YASAR KARATAS $^{1}$, HASAN ESEN $^{3}$, FATIH KESKIN $^{1}$ and ERDAL KALKAN ${ }^{1}$ \\ ${ }^{1}$ Department of Neurosurgery, Meram Faculty of Medicine, Necmettin Erbakan University, Meram, Konya 42080; \\ ${ }^{2}$ Department of Neurosurgery, Ministry of Health, Konya Numune Hospital, Selçuklu, Konya 42060; ${ }^{3}$ Department of \\ Pathology, Meram Faculty of Medicine, Necmettin Erbakan University, Meram, Konya 42080, Turkey
}

Received May 19, 2016; Accepted July 7, 2016

DOI: $10.3892 / \mathrm{mco} .2016 .974$

\begin{abstract}
The present study was designed to evaluate the expression of vascular endothelial growth factor (VEGF), basic fibroblast growth factor (bFGF) and thioredoxin reductase 1 (TrxR1) in glioblastoma multiforme (GBM) with and without intratumoral hemorrhage. Surgically resected human GBM samples from 20 patients who underwent surgery at our institute were extracted from the histopathological specimens and divided into two groups. A total of 10 samples from each type of GBM (World Health Organization grade IV, intratumoral hemorrhage-positive or -negative) were included in each group. VEGF, bFGF and TrxR1 expression was analyzed using immunohistochemistry and the results were compared between groups. VEGF and bFGF immunoreactivity was significantly higher in GBMs containing intratumoral hemorrhage. Furthermore, VEGF, bFGF and TrxR1 immunointensity was significantly higher in GBMs containing intratumoral hemorrhage. Thus, the present study demonstrated a higher VEGF, bFGF and TrxR1 expression in GBMs contain intratumoral hemorrhage, indicatiogn a role of VEGF, bFGF and TrxR1 expression in the promotion of tumoral angiogenesis and tumoral growth by complex mechanisms that require further elucidation.
\end{abstract}

\section{Introduction}

Glioblastoma multiforme (GBM) is the most aggressive and common tumor among primary adult malignant brain tumors, classified as grade IV by the World Health

Correspondence to: Dr Fatih Erdi, Department of Neurosurgery, Meram Faculty of Medicine, Necmettin Erbakan University, Akyokus, Meram, Konya 42080, Turkey

E-mail: mfatiherdi@hotmail.com

Key words: thioredoxin reductase 1, vascular endothelial growth factor, basic fibroblast growth factor, glioblastoma multiforme, intratumoral, hemorrhage
Organization (WHO). GBM is associated with poor survival, despite an aggressive treatment approach including surgery, radiation and chemotherapy (1).

Angiogenesis is required for the growth, invasion and metastasis of cancer cells (2). It has been demonstrated that GBMs exhibit intense angiogenesis, which may be associated with tumor progression and intratumoral hemorrhage (3-5). Angiogenesis is a complex, multistage process that leads to the formation of new blood vessels and depends on the local balance between various molecules that induce or inhibit neovascularization (6). It has been demonstrated that vascular endothelial growth factor (VEGF) plays a pivotal role in the induction of brain tumor angiogenesis (6). Other growth factors, including basic fibroblast growth factor (bFGF), are secreted by the tumor cells, as well as by platelets and, potentially, vascular mesenchymal cells. These factors bind to specific receptors and activate endothelial cells. It has been demonstrated that increased bFGF and VEGF production by cancer cells is directly correlated with tumor angiogenesis and progression (7). Cytosolic thioredoxin ( Trx) also participates in important endothelial cell activities, including migration, proliferation, angiogenesis and apoptosis. The activity of Trx is regulated by thioredoxin reductase 1 (TrxR1) (8).

Evaluating the expression of specific angiogenic and tumorigenic markers is useful for predicting therapeutic responses, grading and prognosis of malignant gliomas (4). Novel treatment options targeting TrxR1 and certain angiogenic molecules are currently under development, with promising results $(4,9,10)$.

The aim of this study was to investigate the association between VEGF, bFGF and TrxR1 expression and intratumoral hemorrhage in GBMs.

\section{Materials and methods}

Patient groups. Surgically resected human GBM samples from 20 patients who underwent surgery at our university (Necmettin Erbakan University, Meram Faculty of Medicine hospital) between 2006 and 2015, were obtained from pathology archives. 
Table I. VEGF, bFGF and TrxR1 expression in groups 1 (with intratumoral hemorrhage) and 2 (without intratumoral hemorrhage).

\begin{tabular}{|c|c|c|c|c|c|}
\hline Groups & $\begin{array}{c}\text { VEGF } \\
\text { immunoreactivity }\end{array}$ & $\begin{array}{c}\text { VEGF } \\
\text { immunointensity }\end{array}$ & $\begin{array}{c}\text { bFGF } \\
\text { immunoreactivity }\end{array}$ & $\begin{array}{c}\text { bFGF } \\
\text { immunointensity }\end{array}$ & $\begin{array}{c}\text { TrxR1 } \\
\text { immunointensity }\end{array}$ \\
\hline $1(n=10)$ & $\begin{array}{l}2.60 \pm 0.60 \\
\quad(2-4)\end{array}$ & $\begin{array}{l}2.30 \pm 0.48 \\
\quad(2-3)\end{array}$ & $\begin{array}{l}2.60 \pm 0.51 \\
\quad(2-3)\end{array}$ & $\begin{array}{l}2.70 \pm 0.48 \\
\quad(2-3)\end{array}$ & $\begin{array}{l}2.70 \pm 0.48 \\
\quad(2-3)\end{array}$ \\
\hline $2(n=10)$ & $\begin{array}{l}1.50 \pm 0.52 \\
\quad(1-2)\end{array}$ & $\begin{array}{c}1.20 \pm 0.78 \\
(0-2)\end{array}$ & $\begin{array}{c}1.70 \pm 0.67 \\
(1-3)\end{array}$ & $\begin{array}{l}1.20 \pm 0.63 \\
(0-2)\end{array}$ & $\begin{array}{c}1.40 \pm 0.69 \\
(0-2)\end{array}$ \\
\hline
\end{tabular}

The values are presented as and mean \pm standard deviation (minimum-maximum). VEGF, vascular endothelial growth factor; bFGF, basic fibroblast growth factor; TrxR1, thioredoxin reductase 1.

The specimens had been fixed in $4 \%$ phosphate-buffered formaldehyde, processed and embedded in paraffin blocks. Histopathological examination, typing and grading of the hematoxylin and eosin-stained slides were performed by an experienced neuropathologist, according to the WHO criteria (11).

The samples included 10 GBM samples containing radiologically and histopathologically confirmed intratumoral hemorrhage (group 1) and 10 GBM samples without evidence of intratumoral hemorrhage (group 2).

None of the patients had been administered radiotherapy, chemotherapy or immunotherapy prior to surgery.

Informed consent for the study was obtained from all patients or their families. Approval for this study was obtained from the local Ethics Committee of Necmettin Erbakan University, Meram Faculty of Medicine.

Immunohistochemistry. Tumor samples previously embedded in paraffin were sectioned $(4 \mu \mathrm{m})$ and mounted onto positively charged slides (Slides Micro Life ${ }^{\circledR}$; Glaswarenfabrik Karl Hecht GmbH \& Co KG, Sondheim, Germany). Immunocytochemistry was then performed using an automated avidin-biotin system (Ventana BenchMark XT; Ventana Medical Systems, Tucson, AZ, USA).

TrxR1 expression was evaluated using a polyclonal antibody (anti-TXNRD1 rabbit polyclonal antibody; 1:200, cat. no. bs-8299R; Bioss, Woburn, MA, USA). VEGF expression was evaluated using a rabbit polyclonal antibody (anti-VEGF; 0.5 mg/ml, cat. no. GTX59912; Gene Tex, Irvine, CA, USA) and bFGF expression was evaluated using a rabbit polyclonal antibody (anti-FGF2; $100 \mu \mathrm{g}$ at $0.5 \mathrm{mg} / \mathrm{ml}$, cat. no. ab126861; Abcam, MA, USA).

The immunointensity of TrxR1 was classified into four groups as follows: 0 , negative; $1+$, weak; $2+$, moderate; and $3+$, strong, as previously reported (12).

VEGF immuno-reactivity was evaluated based on the proportion of immunopositive cells. Five categories were defined as follows: 0 , all negative; $1+,<25 \%$ positive cells; $2+, 25-49 \% ; 3+, 50-74 \%$; and $4+,>75 \%$ positive cells. Immunointensity was also subclassified into four groups as follows: 0, negative; 1+, weak; $2+$, moderate; and 3+, strong $(6,13)$.

bFGF immunoreactivity was evaluated based on the proportions of immunopositive cells. Four categories were defined as follows: 0 , all negative; $1+,<10 \%$ positive cells;
$2+, 10-50 \%$; and $3+,>50 \%$ positive cells. Immunointensity was also subclassified into four groups as follows: 0, negative; 1+, weak; 2+, moderate; and 3+, strong (14).

Statistical analysis. Statistical analyses were performed using SPSS for Windows, version 18.0 (SPSS, Inc., Chicago, IL, USA). Data are expressed as minimum-maximum value, mean \pm standard deviation (SD) and percentage. Statistical analyses were performed using the Chi-square test. A P-value of $<0.05$ was considered to indicate statistically significant differences.

\section{Results}

VEGF, bFGF and TrxR1 expression. Group 1 GBM tissues exhibited statistically significantly higher VEGF and bFGF immunoreactivity $(\mathrm{P}<0.05)$. Group $1 \mathrm{GBM}$ tissues exhibited statistically significantly higher VEGF, bFGF and TrxR1 immunointensity $(\mathrm{P}<0.05)$. Group $1 \mathrm{GBM}$ tissues showed $100 \%, \geq 2+$ immunoreactivity for VEGF and bFGF, and $100 \%$, $\geq 2+$ immunointensity for VEGF, bFGF and TrxR1. Group 2 GBM tissues exhibited $100 \%, \leq 2+$ immunoreactivity for VEGF and bFGF, and $100 \%, \leq 2+$ immunointensity for VEGF, bFGF and TrxR1.

The minimum-maximum and mean \pm SD expression values are summarized in Table I. Representative images of the groups are presented in Fig. 1.

\section{Discussion}

The angiogenetic process is crucial for GBMs, which exhibit intense neovascularization $(1,15)$. Anti-angiogenic targeted therapy is currently being increasingly investigated fort he management of GBM (1).

It has been demonstrated that VEGF and its receptors play a major role in the angiogenesis of malignant gliomas (16). A strong correlation has previously been identified between malignancy in human astrocytic tumors and increased expression of certain fibroblast growth factors, including bFGF (17). The growth and angiogenesis of astrocytomas are positively regulated by bFGF and VEGF, which are secreted by the tumor cells, as well as by platelets and, potentially, by vascular mesenchymal cells $(1,7,18)$. Cytosolic Trx 1 is an isoform of the endogenous antioxidant Trx system, which exerts significant modulating effects on cellular redox status. Growing evidence 


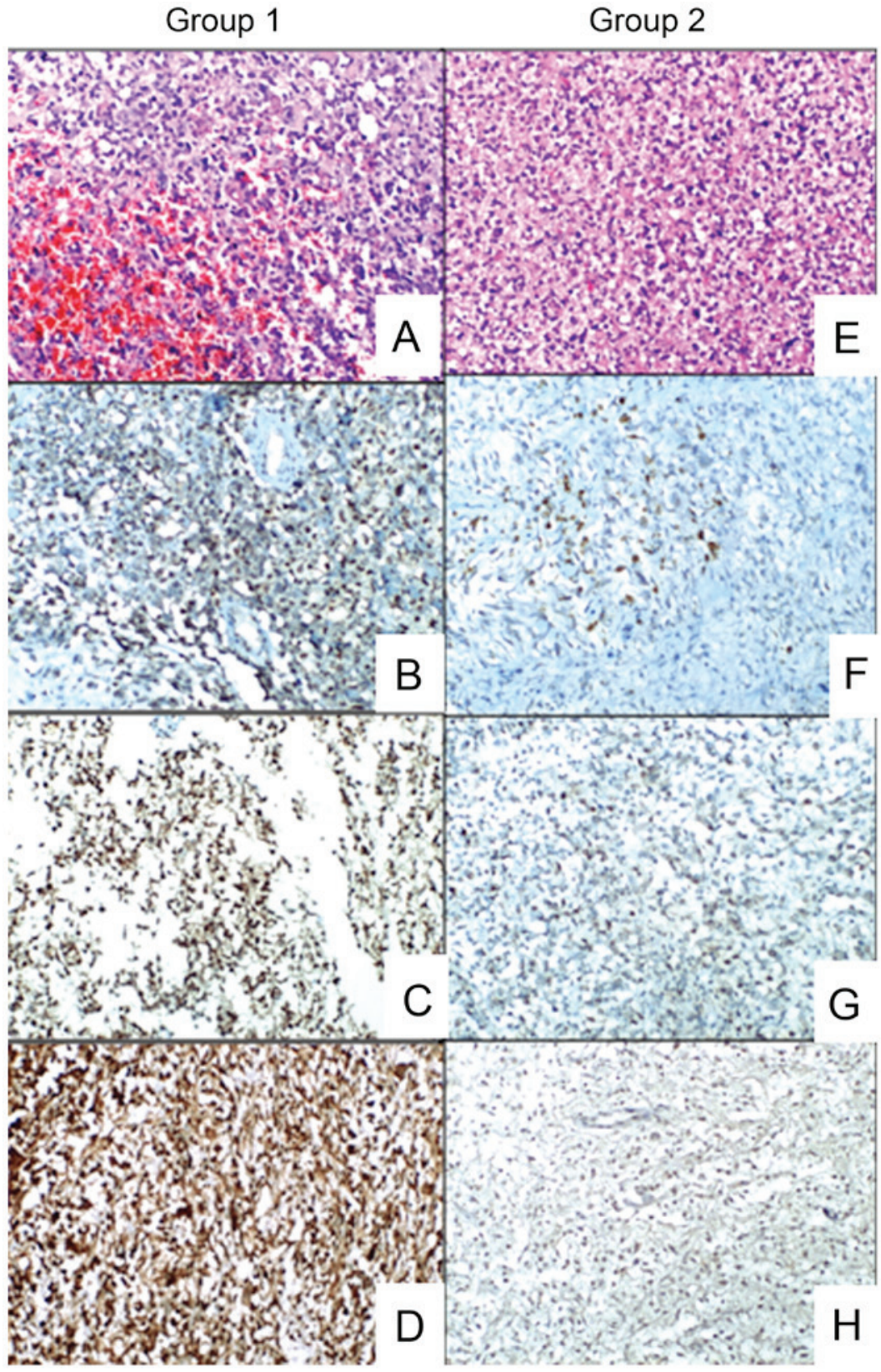

Figure 1. Representative images of the groups. Hematoxylin and eosin (H\&E) staining and immunostaining images for vascular endothelial growth factor (VEGF), basic fibroblast growth factor (bFGF) and thioredoxin reductase 1 (TrxR1). (A) Glioblastoma mutiforme (GBM) sample containing intratumoral hemorrhage (H\&E staining; magnification, x100). (B) Group 1 GBM sample exhibiting strong staining for VEGF (magnification, x100). (C) Group 1 GBM sample exhibiting strong staining for bFGF (magnification, x100). (D) Group 1 GBM sample exhibiting strong staining for TrxR1 (magnification, x100). (E) GBM sample without intratumoral hemorrhage (H\&E staining; magnification, x100). (F) Group 2 GBM sample exhibiting weak staining for VEGF (magnification, x100). (G) Group 2 GBM sample exhibiting weak staining for bFGF (x100). (H) Group 2 GBM sample exhibiting weak staining for TrxR1 (magnification, $\mathrm{x} 100$ ).

suggests that Trx 1 participates in angiogenic signaling pathways by interacting with various transcription factors. The activity of Trx1 is mainly regulated by TrxR1 (8).

The tumoral neovasculature is characterized by increased vessel diameter, length, density and permeability (19). Increased venular and capillary permeability due to enhanced formation of vascular endothelial fenestrations may lead to intratumoral hemorrhage in GBMs $(3,15)$.

Overexpression of VEGF is known to induce tumor-related cyst formation, peritumoral edema formation and intratumoral hemorrhage (3). Cheng et al (15) stereotactically implanted VEGF-overexpressing glioblastoma cells into mouse brains and demonstrated the rapidly developing intracerebral hemorrhage within 60-90 h. Jung et al (20) mentioned the possible role of VEGF overexpression in metastatic brain tumor-related hemorrhage, by achieving rapid vessel growth and breakdown around the tumors. Jin Kim et al (3) indicated the role of VEGF overexpression in tumor-related hemorrhage of pituitary adenomas. However, little is known regarding the role of bFGF and, particularly, TrxR1 in GBMs, although GBMs are associated with a high incidence of spontaneous intratumoral hemorrhage.

In astrocytic tumors, bFGF expression increases with increasing malignancy grade (14). FGF family members, 
including bFGF, possess mitogenic and angiogenic properties, which may explain the role of bFGF in the malignant progression of astrocytic tumors (17). Similarly, the Trx system plays a critical role in the modulation of angiogenesis (8). Trx 1 and VEGF have been found to be overexpressed in a variety of cancers, and increased levels of Trx 1 were correlated with poor prognosis (8). However, there is currently no study in the literature directly demonstrating the expression patterns of bFGF and TrxR1 in tumor-related hemorrhage of GBMs.

In this study, we observed a significant overexpression of VEGF, bFGF and TrxR1 in GBMs containing intratumoral hemorrhage. Our results indicate a role for VEGF, bFGF and TrxR1 in the promotion of angiogenesis. As mentioned previously, enhanced angiogenesis may lead to intratumoral hemorrhage (18) and tumoral growth (20) by complex mechanisms that have not yet been fully elucidated. To the best of our knowledge, our results represent the first evidence on the expression variations of VEGF, TrxR1 and bFGF in GBMs with or without intratumoral hemorrhage.

There were certain limitations to this study, including the relatively small sample size and semiquantitative assessment method by immunohistochemical staining. Futher research, using quantitative reverse transcription polymerase chain reaction with immunostaining, may yield more sensitive results.

\section{References}

1. Zhang M, Ye G, Li J and Wang Y: Recent advance in molecular angiogenesis in glioblastoma: The challenge and hope for anti-angiogenic therapy. Brain Tumor Pathol 32: 229-236, 2015.

2. Arnér ES and Holmgren A: The thioredoxin system in cancer. Semin Cancer Biol 16: 420-426, 2006.

3. Jin Kim Y, Hyun Kim C, Hwan Cheong J and Min Kim J: Relationship between expression of vascular endothelial growth factor and intratumoral hemorrhage in human pituitary adenomas. Tumori 97: 639-646, 2011.

4. Gatson NN, Chiocca EA and Kaur B: Anti-angiogenic gene therapy in the treatment of malignant gliomas. Neurosci Lett 527: 62-70, 2012.

5. Stiver SI: Angiogenesis and its role in the behavior of astrocytic brain tumors. Front Biosci 9: 3105-3123, 2004.

6. Hara A and Okayasu I: Cyclooxygenase-2 and inducible nitric oxide synthase expression in human astrocytic gliomas: Correlation with angiogenesis and prognostic significance. Acta Neuropathol 108: 43-48, 2004.
7. Jang FF, Wei W and De WM: Vascular endothelial growth factor and basic fibroblast growth factor expression positively correlates with angiogenesis and peritumoural brain oedema in astrocytoma. J Ayub Med Coll Abbottabad 20: 105-109, 2008.

8. Dunn LL, Buckle AM, Cooke JP and Ng MK: The emerging role of the thioredoxin system in angiogenesis. Arterioscler Thromb Vasc Biol 30: 2089-2098, 2010.

9. Yagublu V, Arthur JR, Babayeva SN, Nicol F, Post S and Keese M: Expression of selenium-containing proteins in human colon carcinoma tissue. Anticancer Res 31: 2693-2698, 2011.

10. Holash J, Maisonpierre PC, Compton D, Boland P, Alexander CR, Zagzag D, Yancopoulos GD and Wiegand SJ: Vessel cooption, regression, and growth in tumors mediated by angiopoietins and VEGF. Science 284: 1994-1998, 1999.

11. Louis DN, Ohgaki H, Wiestler OD, Cavenee WK, Burger PC, Jouvet A, Scheithauer BW and Kleihues P: The 2007 WHO classification of tumours of the central nervous system. Acta Neuropathol 114: 97-109, 2007.

12. Haapasalo H, Kyläniemi M, Paunul N, Kinnula VL and Soini Y: Expression of antioxidant enzymes in astrocytic brain tumors. Brain Pathol 13: 155-164, 2003.

13. El-Sayed M and Taha MM: Immunohistochemical expression of cycloxygenase- 2 in astrocytoma: Correlation with angiogenesis, tumor progression and survival. Turk Neurosurg 21: 27-35, 2011.

14. Torp SH and Alsaker M: Ki-67 immunoreactivity, basic fibroblastic growth factor (bFGF) expression, and microvessel density as supplementary prognostic tools in low-grade astrocytomas. An immunohistochemical study with special reference to the reliability of different Ki-67 anti. Pathol Res Pract 198: 261-265, 2002.

15. Cheng SY, Nagane M, Huang HS and Cavenee WK: Intracerebral tumor-associated hemorrhage caused by overexpression of the vascular endothelial growth factor isoforms VEGF121 and VEGF165 but not VEGF189. Proc Natl Acad Sci USA 94: 12081-12087, 1997.

16. Plate KH and Risau W: Angiogenesis in malignant gliomas. Glia 15: 339-347, 1995.

17. Morrison RS, Yamaguchi F, Saya H, Bruner JM, Yahanda AM, Donehower LA and Berger M: Basic fibroblast growth factor and fibroblast growth factor receptor I are implicated in the growth of human astrocytomas. J Neurooncol 18: 207-216, 1994.

18. Bian XW, Du LL, Shi JQ, Cheng YS and Liu FX: Correlation of bFGF, FGFR-1 and VEGF expression with vascularity and malignancy of human astrocytomas. Anal Quant Cytol Histol 22: 267-274, 2000

19. Harrigan MR: Angiogenic factors in the central nervous system. Neurosurgery 53: 639-660; discussion 660-661, 2003.

20. Jung S, Moon KS, Jung TY, Kim IY, Lee YH, Rhu HH, Sun HS, Jeong YI, Kim KK and Kang SS: Possible pathophysiological role of vascular endothelial growth factor (VEGF) and matrix metalloproteinases (MMPs) in metastatic brain tumor-associated intracerebral hemorrhage. J Neurooncol 76: 257-263, 2006. 